\title{
Prognostic significance of histopathological tumor regression after neoadjuvant chemotherapy in esophageal adenocarcinomas
}

\author{
Rupert Langer $^{1,5}$, Katja Ott ${ }^{2,5}$, Marcus Feith ${ }^{3}$, Florian Lordick ${ }^{4}$, Jörg-Rüdiger Siewert ${ }^{3}$ and \\ Karen Becker ${ }^{1}$ \\ ${ }^{1}$ Institute of Pathology, Technische Universität München, München, Germany; ${ }^{2}$ Department of Surgery, \\ Universität Heidelberg, Heidelberg, Germany; ${ }^{3}$ Department of Surgery, Klinikum Rechts der Isar, Technische \\ Universität München, München, Germany and ${ }^{4}$ Department of Medical Oncology, National Center for Tumor \\ Diseases (NCT), Heidelberg, Germany
}

\begin{abstract}
We evaluated histomorphological findings in 92 surgical resection specimens of locally advanced esophageal adenocarcinomas after neoadjuvant cisplatin-based chemotherapy. Tumor response to neoadjuvant chemotherapy was determined using a system encompassing three tumor regression grades based on the estimation of the percentage of residual tumor tissue of the primary tumor site in relation to the macroscopically identifiable previous tumor bed. The significance of this system was validated by correlation of the tumor regression grades with the corresponding clinicopathological characteristics and patient survival. Seven patients $(7 \%)$ had complete tumor regression (grade tumor regression grade 1$)$, 48 patients $(52 \%)$ had subtotal or partial tumor regression (tumor regression grade 2: 1-50\% residual tumor), and 37 patients $(40 \%)$ had minimal or no regression (tumor regression grade 3: $>\mathbf{5 0} \%$ residual tumor). Tumor regression was significantly associated with posttreatment complete tumor resection status (UICC R0 status; $P=0.016$ ), tumor category (UICC pT category; $P<0.001)$, and with the absence of either lymph node metastases $(P=0.001)$ or lymphatic invasion $(P<0.001)$. Survival analysis showed a significant prognostic relevance of the applied regression system in univariate $(P<0.001)$ and multivariate analyses as a single independent factor $(P=0.024)$. We conclude that the effect of preoperative chemotherapy in esophageal adenocarcinomas can be assessed by the determination of histological tumor regression, providing highly valuable prognostic information, which may even exceed the prognostic impact of the current TNM classification of these tumors. Therefore, we strongly recommend the implementation of a standardized tumor regression grading system in pathological reports of esophageal adenocarcinomas treated by neoadjuvant chemotherapy.
\end{abstract}

Modern Pathology (2009) 22, 1555-1563; doi:10.1038/modpathol.2009.123; published online 2 October 2009

Keywords: adenocarcinoma; esophagus; regression; classification; chemotherapy

Esophageal adenocarcinoma is increasing rapidly in frequency. ${ }^{1,2}$ At present, it is the seventh leading cause of cancer-related death in the western world. ${ }^{3}$ The most common therapeutic approach to treat locally

Correspondence: Dr R Langer, MD, Institute of Pathology, Klinikum Rechts der Isar, Technische Universität München, Trogerstrasse 18, D-81675 München, Germany.

E-mail: rupert.langer@lrz.tu-muenchen.de

${ }^{5}$ These authors contributed equally to this work.

Received 2 March 2009; revised 8 July 2009; accepted 20 July 2009; published online 2 October 2009 advanced esophageal adenocarcinomas (Barrett's carcinomas) is a multimodal treatment with preoperative Cisplatinum/5-fluorouracil-based chemotherapy or radiochemotherapy, followed by resection. ${ }^{4-6}$

Recent publications have suggested that this treatment concept increases patient survival when compared with surgery alone, ${ }^{7}$ despite the fact that in $\sim 50 \%$ of the patients, no measurable response is achieved. ${ }^{8}$ In clinical practice, it is accepted that patients who respond to preoperative treatment have significantly improved survival than do those who do not respond. However, no generally 
accepted standardized clinical or histopathological response evaluation system has been established so far. Histological tumor regression after chemotherapy is believed to be an important objective parameter and has been shown to have prognostic value in several studies. ${ }^{9-11}$ However, the determination of histopathological tumor regression in these studies lacks comparability because tumors are treated differently and specimens are processed and graded differently with respect to regression.

In this study, we present our diagnostic procedure for resection specimens of esophageal adenocarcinomas after neoadjuvant chemotherapy with a standardized macroscopical and histological workup, considering and highlighting the specific characteristics of tumors after treatment. For evaluating tumor regression, we applied a scoring system encompassing three tumor regression grades. ${ }^{12}$ The clinical and prognostic value of this tumor regression grading system is shown by the correlation with clinicopathological parameters and patient outcome, thus warranting the recommendation for the implementation of a standardized tumor regression grading system for an accurate and prognostic relevant classification of tumors after chemotherapy.

\section{Materials and methods}

\section{Patients}

A total of 92 patients were enrolled in this study. Each patient had locally advanced (cT3/4cN0-1cM0/1a) esophageal adenocarcinoma, (adenocarcinoma of the esophagogastric junction type I according to Siewert and Stein ${ }^{13}$ ), which was proven by biopsy, and underwent neoadjuvant chemotherapy between 1994 and 2002 at the Department of Surgery of the Technische Universität München. Pre-therapeutic staging procedures for confirming cT3/4 categorization included endoscopy, endoscopic ultrasound, and computed tomography of the chest and abdomen. None of the patients received additional radiation therapy. Patients who had undergone previous chemotherapy, radiotherapy, or endoscopic laser therapy were not included. The study protocols of neoadjuvant treatment were approved by the Institutional Review Board at the Technische Universität München.

Patients were treated by at least one cycle of concurrent cisplatin-based chemotherapy, followed by either esophagectomy or gastrectomy. Patient follow-up assessment was performed every 3 months for the first year and at 6-month intervals thereafter. Overall survival was calculated from the first day of chemotherapy application.

\section{Morphological Evaluation of Tumor Regression}

The 92 resection specimens were evaluated according to a standardized protocol. After photographic documentation, a 1:1 photocopy of the resection
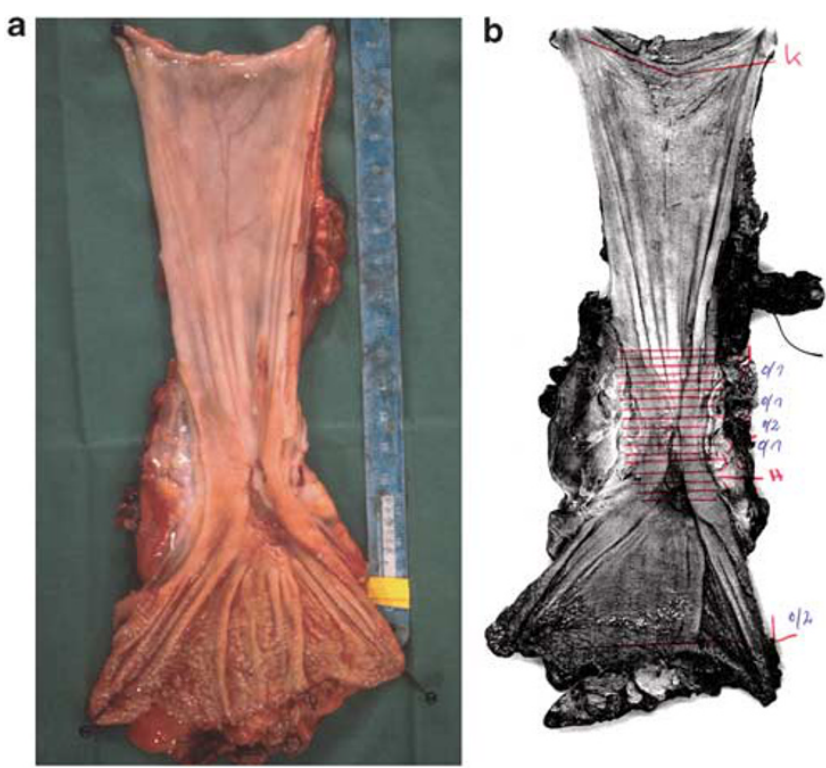

Figure 1 Esophagectomy specimen. Macroscopy (a) and corresponding photocopy work-up (b) of a resection specimen of an esophageal adenocarcinoma showing complete serial sectioning of the tumor bed after neoadjuvant chemotherapy.

specimen was made (Figure 1). The entire macroscopic identifiable tumor or the area with scarring indicating the site of previous tumor (the tumor bed) was measured and cross-sectioned serially at $0.5-\mathrm{cm}$ intervals and embedded completely (median: 11.2 sections; range: 9-18). Tissue sections were stained with hematoxylin and eosin. Selected slides were additionally stained with Elastic van Gieson stain to distinguish between tumor desmoplasia and scarring, as a result of chemotherapy showing textural disarray different from desmoplasia. Periodic acidSchiff stains and immunohistochemistry for cytokeratins (one slice) helped to distinguish signet ring cells from histiocytes, which was necessary in four cases. In cases of initial failure to detect residual tumor cells, additional three step sections were performed to confirm complete tumor response. The resection margins and lymph node stations were evaluated separately.

The tumor specimens were typed and graded according to the guidelines of the WHO and were classified according to the Lauren type, analogous to the classification of gastric adenocarcinomas. The extent of the tumor (TNM classification) and the completeness of the resection (R-status) were determined according to the UICC guidelines. Tumor involvement of lymphatic vessels was classified as either absent or present. The grading of tumor regression in response to chemotherapy was based on an estimation of the percentage of residual tumor tissue in relation to the macroscopically identifiable tumor bed of the primary site of the tumor. The slides were reviewed separately by two pathologists (KB and RL). In case of disagreement regarding tumor regression grading, both pathologists 
Table 1 Histopathological tumor regression grading

Tumor regression Description grade

$\begin{array}{ll}1 & \begin{array}{l}\text { No residual tumor/tumor bed+chemotherapy } \\ \text { effect }\end{array} \\ & 1-50 \% \text { residual tumor/tumor } \\ & \text { bed+chemotherapy effect } \\ & >50 \% \text { residual tumor/tumor } \\ \text { bed } \pm \text { chemotherapy effect } & \end{array}$

${ }^{a}$ Specific chemotherapy-associated alterations (chemotherapy effects): scarry fibrosis of the inner, luminal parts of the tumor bed, histiocytic foamy cells, acellular mucus lakes, and reactive vascular changes.

reviewed the specimen under a double-headed microscope and reached a consensus diagnosis. The following system of tumor regression grades was used: grade 1 , complete ( $0 \%$ residual tumor), grade 2, subtotal and partial tumor regression ( $1-50 \%$ residual tumor per tumor bed), and grade 3 , minimal or no tumor regression ( $>50 \%$ residual tumor per tumor bed) (see also Tables 1 and 2).

For initial raw data analysis, subtotal tumor regression (1-10\% residual tumor per tumor bed) and partial tumor regression $(11-50 \%$ residual tumor per tumor bed) were evaluated separately. On the basis of the observations that failed to render any significant prognostic difference between these two groups (given as Supplementary Data), these subgroups were subsumed for definite analysis in the final regression system.

\section{Statistics}

Associations between tumor regression grades and pathological features were evaluated by $\chi^{2}$ tests and Fisher's exact tests. Survival analysis was performed using Kaplan-Meier estimates, log-rank tests, and Cox's proportional hazards regression analysis. All tests were two-sided, and the significance level was set at 5\%. For all statistical procedures, SPSS 16 software (SPSS, Chicago, IL, USA) was used.

\section{Results}

\section{Patients}

The patients had a mean age of 56.6 years (range: 25-79 years). There were 6 women and 86 men. A total of 61 patients received chemotherapy with cisplatin/5-fluorouracil/Leucovorine, with 19 of those individuals receiving additional paclitaxel. Seventeen patients received chemotherapy with etoposide/adriamycin/cisplatin. Fourteen patients received some other cisplatin-based chemotherapy. Esophagectomy was performed in 59 patients, and gastrectomy (transhiatal extended) was performed in 33 patients.
Table 2 Histopathological work-up of neoadjuvant-treated esophageal adenocarcinomas

Photographic documentation

Photocopy of resection specimen (orientation and documentation of blocks and of histologically proven residual tumor)

Macroscopic description; tumor size (three-dimensional), distance to resection margins

Work-up

Inking of the deep resection margin

Complete embedding of the macroscopically identifiable tumor bed, orientated from proximal to distal in 0.5-cm levels.

Hematoxylin/eosin, Periodic acid-Schiff, Elastic van Gieson staining

If no residual tumor: another three step sections to confirm complete response

Resection margins oral, aboral

Additional areas suspicious for Barrett's mucosa

Lymph node stations. Immunohistochemistry (pan-Cytokeratin) if ypNo

Pathological report should include

UICC ypTNML status

UICC R-status

Grading, typing (according to WHO and analogous to Lauren)

Histopathological tumor regression grade (grade 1, 2, 3)

\section{Histopathological Classifications}

Preoperative tumor biopsies showed adenocarcinomas, analogous to the Lauren classification of intestinal type in 65 cases (70\%), of diffuse type in 6 cases ( $8 \%)$, and of mixed type in 21 cases $(22 \%)$. According to the current WHO classification, most cases (68 cases; 73\%) were of the tubular/papillary type and 11 cases $(12 \%)$ were mucinous. The remaining cases contained three signet-cell carcinomas, one adenosquamous carcinoma, one carcinoma with neuroendocrine differentiation, and eight undifferentiated or solid carcinomas. Tumor grading was G1 in one case (1\%), G2 in 31 cases (33\%), G3 in 48 cases (52\%), and G4 in 12 cases (14\%).

Postoperative tumor categories were ypT0 in 7 cases (8\%), ypT1 in 9 cases (10\%), and ypT2 in 19 cases (21\%); therefore, a tumor down-categorization from cT3 to ypT0-T2 was observed in 35 cases $(38 \%)$. A total of 57 tumors (62\%) were of the ypT3 category. Lymph node metastases were present in 57 cases $(62 \%)$. The mean number of lymph nodes resected was 25.6 (range: 11-66) and the mean number of lymph node metastases was 4.6 per case (range: 0-30). Lymphatic invasion was detected in 58 cases $(63 \%)$. In all, 63 patients (69\%) had complete tumor resection (R0-resection). Of the 29 patients $(32 \%)$ with incomplete tumor resection, 16 had positive circumferential margins (17\%), 3 had positive circumferential and distal margins (4\%), and 2 had positive circumferential and proximal margins $(2 \%)$. In three patients, there was the presence of lymphatic vessel invasion and/or lymph node metastases within the resection margins. In five patients, the circumferential margins were highly suspicious for being positive (RX; $5 \%$ ). 


\section{Chemotherapy-Related Histomorphological Findings}

Histomorphological features, which were generally characteristic of chemotherapeutic treatment, included marked, partly transmural fibrosis with occasional scattered residual tumor glands at the periphery of the tumor bed (Figure 2a-c and h), foamy histiocytes (Figure 2e-g), acellular mucus lakes (Figure 2d), and vascular changes, inclu- ding endangiitis obliterans and organizing thrombi (Figure 2i). With regard to the centrifugal tumor regression, areas close to the lumen consisted of tumor-free fibrosis in the central, superficial portion of the tumor bed with discontinuous islands of residual tumor in deeper, more peripheral areas. Using the regression system described above, seven patients were classified as tumor regression grade 1, which could be confirmed in all cases by step
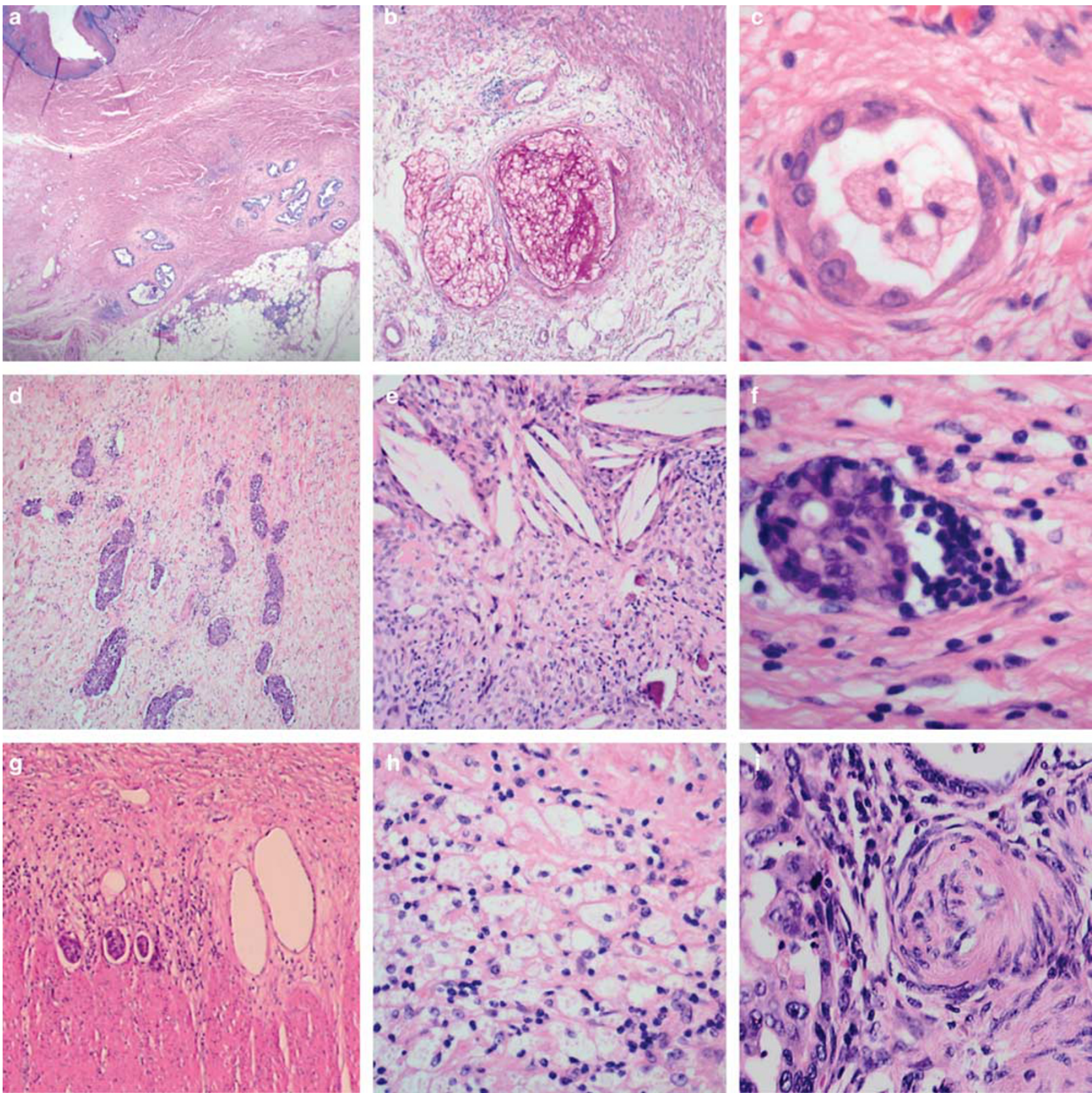

Figure 2 Histopathological findings in esophageal adenocarcinoma after neoadjuvant chemotherapy: partly transmural fibrosis with occasional scattered residual tumor glands (a) (period acid-Schiff, $\times 5$ ), (c) (hematoxylin/eosin, $\times 40)$, and (d) (hematoxylin/eosin, $\times 10)$. Fibrosis and the presence of lymphatic vessel invasion (g) (hematoxylin/eosin, $\times 10$ ). Acellular mucus lakes (b) (period acid-Schiff, $\times 10$ ). Resorptive changes with cholesterol deposits, dystophic calcifications and foamy histiocytes (e) (hematoxylin/eosin, $\times 15)$. Foamy histiocytes $(\mathbf{h})$. (hematoxylin/eosin, $\times 20$ ). Tumor gland with a lymphocytic infiltrate $\mathrm{F}($ hematoxylin/eosin, $\times 40)$. Vascular changes, including endangiitis obliterans and organizing thrombi I (hematoxylin/eosin, $\times 20$ ). 
sectioning as described above. A total of 49 patients had subtotal or partial tumor regression (tumor regression grade 2), and 37 patients had minimal or no regression (tumor regression grade 3 ) (Table 3 ).

\section{Correlations Between Histological Tumor Regression and Histopathological Classifications}

Tumor differentiation grading and histological tumor typing based on either the Lauren classification system or the WHO classification system showed no association with tumor regression $(P=\mathrm{NS})$. ypT category was associated with tumor regression. In particular, there was a significant association between tumor regression grade 1-2 and tumor downstaging to ypT0-2, whereas more than half of the patients with ypT3 were histologically minimal or nonresponders $(P<0.001)$. Tumor regression was also associated with the absence of lymph node metastases (ypN category; $P=0.001$ ) and with the absence of lymphatic invasion $(P<0.001)$. Finally, patients with minimal or no tumor regression more frequently failed to have complete tumor resection (R0; $P=0.016$ ) (see Table 3).

\section{Survival Data}

The minimum follow-up time for surviving patients was 6 years. Five patients were lost to follow-up. The 5-year survival rate was $42 \%$, and the median survival period was 36 months (range: 5-144 months).

All seven patients with complete tumor regression (tumor regression grade 1) are still alive. The median survival age was 51 months (range: 6-143 months) for patients with tumor regression grade 2 and 16 months (range: 5-104 months) for those with tumor regression grade 3 .

UICC ypT category $(P<0.001)$, UICC ypN category $(P<0.001)$, lymphatic vessel invasion $(P<0.001)$, UICC R-status $(P=0.004)$, and tumor regression grade $(P<0.001)$ were found to be significantly correlated with survival, by univariate analysis. Higher tumor differentiation grade (G1/G2 vs G3/ G4) was also associated with prolonged survival $(P=0.017)$ (Figures 3 and 4$)$.

Multivariate analysis that included ypT, ypN, lymphatic invasion, resection status, and tumor regression grade showed histopathological tumor regression as having a single independent prognostic significance of $P=0.024$ (see also Table 4). In addition, patients with ypT3 tumors and a tumor regression grade 2 had significantly better outcome with a median survival of 40 months (range: 8-113) than did patients with tumor regression grade 3 in the same ypT category with a median survival of 12 months (range: $5-105 ; P=0.024$ ). These results did not change significantly when excluding patients with known distant metastases (M1a; $n=13$ ) at the time of surgery.
Table 3 Histopathological tumor regression grading and clinicopathologic features

\begin{tabular}{|c|c|c|c|c|c|}
\hline & \multirow[b]{2}{*}{$\underset{\text { (total) }}{\mathrm{n}}$} & \multicolumn{3}{|c|}{$\begin{array}{l}\text { Histopathological tumor } \\
\text { regression grade }\end{array}$} & \multirow[b]{2}{*}{ P-value } \\
\hline & & $\begin{array}{l}\text { Grade } 1 \\
\text { (n) }\end{array}$ & $\begin{array}{l}\text { Grade } 2 \\
\text { (n) }\end{array}$ & $\begin{array}{l}\text { Grade } 3 \\
\text { (n) }\end{array}$ & \\
\hline \multicolumn{6}{|l|}{ Tumor grading } \\
\hline G1-2 & 32 & 5 & 18 & 9 & NS \\
\hline G3-4 & 60 & 2 & 30 & 28 & \\
\hline \multicolumn{6}{|c|}{ Lauren's classification } \\
\hline $\begin{array}{l}\text { Intestinal } \\
\text { type }\end{array}$ & 65 & 6 & 26 & 23 & NS \\
\hline Mixed type & 21 & 0 & 11 & 10 & \\
\hline Diffuse type & 6 & 0 & 2 & 4 & \\
\hline \multicolumn{6}{|c|}{ UICC урT category } \\
\hline урто & 7 & 7 & 0 & 0 & \\
\hline ypT1 & 9 & 0 & 9 & 0 & $<0.001 *$ \\
\hline урТ2 & 19 & 0 & 15 & 4 & \\
\hline урТ3 & 57 & 0 & 24 & 33 & \\
\hline \multicolumn{6}{|c|}{ UICC ypN category } \\
\hline ypNo & 35 & 4 & 25 & 6 & 0.001 \\
\hline ypN1 & 57 & 3 & 23 & 31 & \\
\hline \multicolumn{6}{|c|}{ UICC урL category } \\
\hline ypL0 & 35 & 7 & 22 & 6 & $<0.001$ \\
\hline ypL1 & 57 & 0 & 26 & 31 & \\
\hline \multicolumn{6}{|l|}{ Resection status } \\
\hline R0 & 63 & 7 & 38 & 18 & 0.016 \\
\hline R1-2 & 29 & 0 & 10 & 19 & \\
\hline
\end{tabular}

$P$-values are given for overall comparison of cross-tabs $\left(\chi^{2}\right.$ test or Fisher's exact test). ${ }^{*} P=0.001$ when excluding patients with ypT0/ tumor regression grade 1 tumors.

\section{Discussion}

Neoadjuvant (radio)chemotherapy is being increasingly used in the treatment of locally advanced esophageal adenocarcinomas to improve patient outcome. This approach may increase local resectability rates and eliminate distant micrometastases. ${ }^{4,5,14}$ Although the benefits of this concept still remain controversial, the results of the recent randomized British Phase III study, which showed a survival benefit for patients with perioperative chemotherapy when compared with surgery alone, ${ }^{4}$ have recently been confirmed in clinical practice. ${ }^{6}$

In this study, we investigated the importance of a standardized and complete work-up of tumor resection specimens to accurately determine the histopathological regression of locally advanced esophageal adenocarcinomas after neoadjuvant chemotherapy. We used a homogenous collection of patients to achieve standardized conditions for pathological analysis. The most common neoadjuvant treatment of esophageal adenocarcinomas at our institution was cisplatin-based chemotherapy (without radiation). ${ }^{5,14,15}$ Therefore, patients who were treated with additional radiation for 

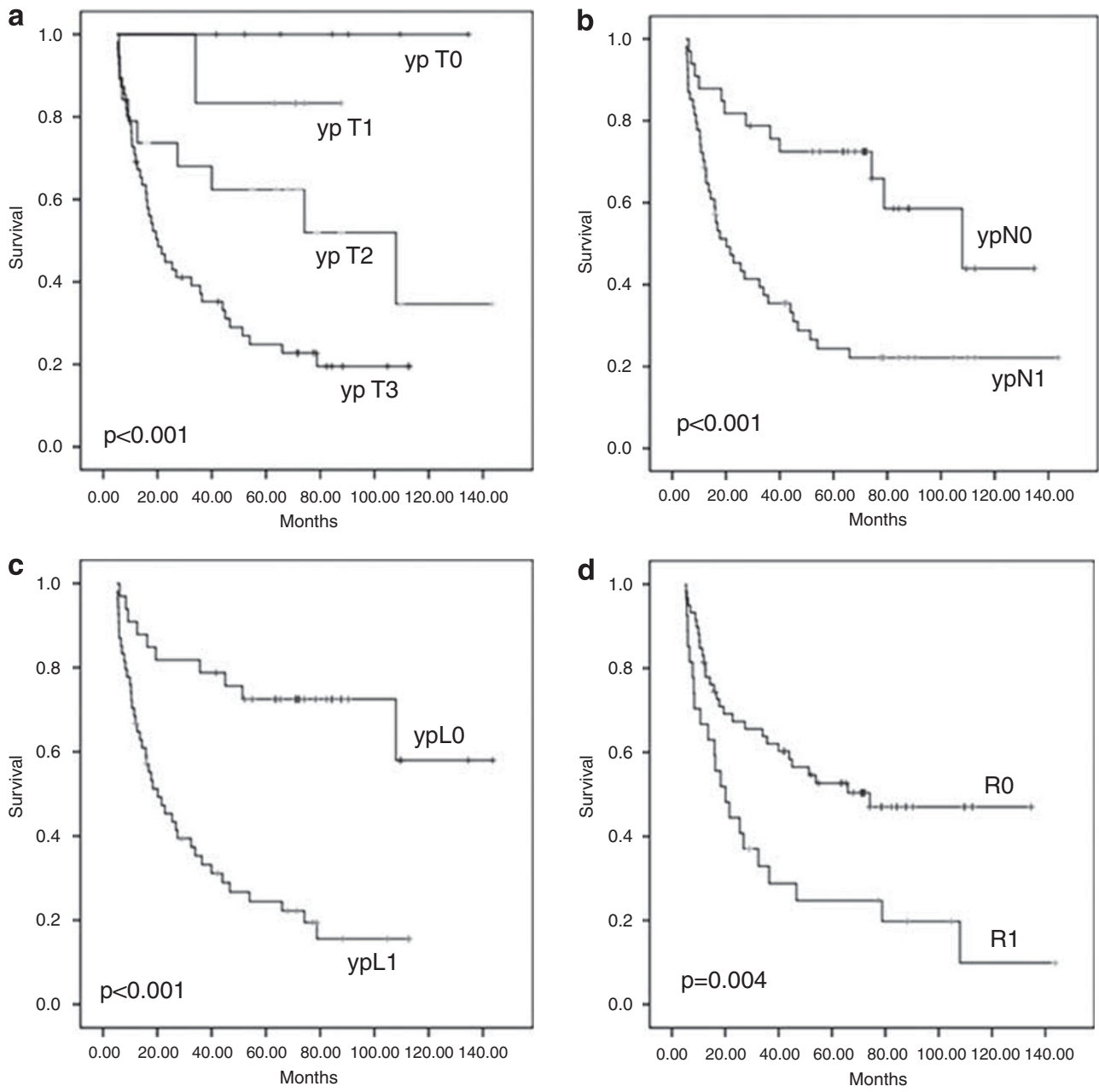

Figure 3 Clinicopathological features of (a) ypT category, (b) ypN category, (c) lymphatic vessel invasion, and (d) resection status) and survival after chemotherapy and surgery are represented.

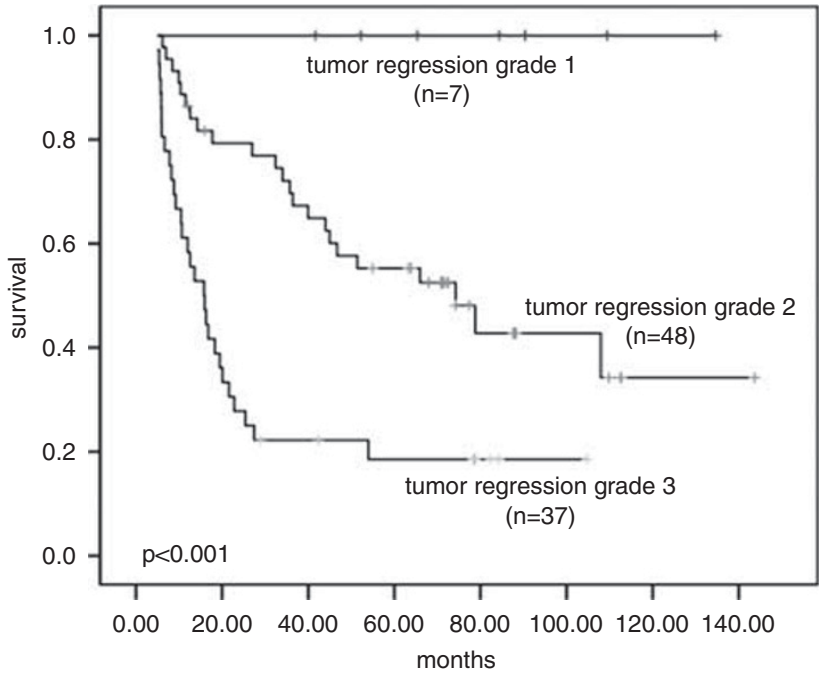

Figure 4 Tumor regression and survival after chemotherapy and surgery (grade 1: 7 patients; grade 2: 48 patients; grade 3: 37 patients). exceptional reasons were not included, nor were those with therapy discontinuation before completing one full cycle of chemotherapy included.

Histopathological examination of the posttreatment resection specimens revealed morphological changes that have been described previously as being typical of chemotherapy-induced changes in adenocarcinomas of the gastrointestinal tract. These changes generally represent a pattern of subacute inflammation, and in addition decreased tumor cell density, acellular mucus lakes, foamy histiocytes, fibrosis (especially in the submucosa and muscularis propria), dissolution of glands, giant cells, and cytological changes, such as apoptotic bodies and intracellular vacuoles. ${ }^{8,12,16}$ Furthermore, tumor regression showed a centrifugal pattern with tumorfree fibrosis in the central and superficial, luminal portions of the tumor bed and residual tumor in deeper areas of the periphery; although tumor regression was associated with tumor downstaging of the ypT category in more than half of the patients, 
Table 4 Multivariate analysis for survival, including ypT category, ypN category, ypL category (lymphatic vessel invasion), tumor resection status, and tumor regression grading

\begin{tabular}{lcr}
\hline Factor & EXP (B) & 95\% CI \\
\hline UICC ypT classification & 1.63 & $0.86-3.08$ \\
UICC ypN classification (lymph node involvement absent vs present) & 1.85 & $0.93-3.68$ \\
UICC ypL classification (lymphatic vessel invasion absent vs present) & 1.96 & $0.90-4.27$ \\
Tumor regression grade & 1.99 & $1.10-3.60$ \\
Tumor resection status & 1.23 & $0.71-2.14$
\end{tabular}

CI, confidential interval.

the remaining tumors with a partial regression (tumor regression grade 2) still had a ypT3 category. These findings confirm the need to work-up the whole tumor bed to detect scattered tumor residuals that are important for determining an accurate tumor category and regression classification. In addition, these observations may explain differences between clinical and histopathological estimation of tumor regression, given that superficially tumor-free areas after chemotherapy may actually harbor larger amounts of residual tumors in deeper areas of the tumor bed.

For determining tumor regression after chemotherapy treatment, we applied a three-score tumor regression system for the primary site of the tumor which was originally introduced for gastric cancer with slight modifications. ${ }^{12}$ We have successfully applied a similar scoring system to esophageal squamous carcinomas ${ }^{17}$ and rectal cancer, ${ }^{18}$ and have shown an objective prognostic impact for total or subtotal tumor regression after neoadjuvant (radio)chemotherapy in these tumors. Three-tiered regression scoring systems have also been used for larger retrospective studies by others. ${ }^{19,20}$ Moreover, a high inter-observer reliability for those systems has been shown, with those systems equally applicable to both squamous cell carcinoma and adenocarcinoma. ${ }^{21}$ After 15 years of diagnostic experience, we favor this scoring system in clinical practice over the widely used regression system described by Dunne et $a l^{16}$ and Mandard et $a{ }^{2},{ }^{22}$ which includes five tumor regression grades, based on the percentage of residual tumor in relation to therapy-induced fibrosis. A scoring system of three grades seems to be more easily implemented, more reproducible, and stronger and clearer with regard to prognostic impact.

Using this tumor regression system, we showed a significant association between the degree of tumor regression and postoperative ypT, ypN, and ypL (lymphatic vessel invasion) classification, as well as between the tumor regression grade and the rate of total tumor resection in esophageal adenocarcinomas. Similar results showing a clear survival benefit for patients with complete tumor response have been obtained by others in studies focusing on esophageal adeno and squamous cell carcinomas after (radio)chemotherapy. ${ }^{9,11,17,19,20,23-25}$ In this context, we would like to point out the important role of the presence of lymphatic vessel invasion in our study, as it had a similar prognostic impact as lymph node involvement. This is in contrast to what has been observed in primary resected esophageal carcinomas, in which lymphatic invasion has been shown to be of minor prognostic importance when compared with squamous cell carcinomas. ${ }^{26,27} \mathrm{How}-$ ever, some differences from other studies in esophageal adenocarcinomas can be noted, most of which can be accounted for the reports of complete tumor regression rates of $>20 \% .^{9,19}$ This may be because of the fact that additional radiation may enhance the rate of local total tumor regression. In addition, it is also possible that these discrepancies in the rates of 'complete' tumor regression are actually due to a histological work-up of the resection specimens that is less extensive than our processing standards. In addition, reported clinical data for complete responders after radiochemotherapy show 5-year survival rates of approximately $60-70 \%,{ }^{20,23}$ which is lower than those of our study, in which all patients with complete tumor response are still alive.

The most striking result of our study is the fact that our tumor regression system seems to have a stronger prognostic impact as the current TNM system for tumors after neoadjuvant chemotherapy. Results of other studies of both esophageal squamous cell carcinomas and adenocarcinomas treated by preoperative radiochemotherapy also indicate that the degree of histopathological tumor regression after preoperative treatment may be a better predictor of clinical outcome than the above-mentioned classification systems. ${ }^{20,23}$ In our study, we confirm these findings for esophageal adenocarcinomas treated by chemotherapy without radiation by univariate and multivariate survival analyses, showing the degree of tumor regression after chemotherapy as the only significant independent prognostic factor compared with the ypTNM classification and the resection status of tumors. Moreover, one major point seems to be the observation that in the same group of patients with ypT3 category tumors, there is a significant prognostic difference between patients with tumor regression grade 2 and those with tumor regression grade 3 . This strongly highlights the importance of even a partial tumor regression. 
In most other studies-which are mentioned above-the prognostic impact of partial tumor regression remains unclear, especially given that there is no consistent definition of a partial response between various studies, nor in the work-up of the resection specimens. Given that all seven patients in this study with complete tumor regression are still alive, complete tumor regression after chemotherapy is the most favorable prognostic factor. However, our results strongly emphasize the importance of even a partial tumor regression for patient survival, such that there is a separate intermediate prognostic category that can be used for these patients.

Taken together, our findings may help to better define clear subgroups of patients with a potential differential clinical course that may have a benefit from individualized risk-adopted aftercare and additional therapeutic considerations. Furthermore, our results may be of high importance for scientific research, in view of many studies that rely on having accurately characterized patient cohorts. One example would be studies that aim at identifying any molecular markers that can predict the response to (radio)chemotherapy, especially in the case of esophageal adenocarcinomas, for which there is still a significant number of patients who do not respond to neoadjuvant (radio)chemotherapy, as shown in this and other studies. ${ }^{8,9,23}$ A valid and reproducible classification of tumor response should be an indispensable and substantial tool in research aimed at identifying and verifying molecular markers for response prediction.

In conclusion, standardized determination of histopathological tumor regression provides valid prognostic and clinical correlations. Not only does this classification have an immediate impact on pathological and clinical practice, especially when considering risk-adopted therapeutic decisions, but also for furthers research in the field of response prediction and individualized treatment of esophageal cancer.

\section{Acknowledgement}

This work is dedicated to Heinz Höfler, the Head of the Department of Pathology of the Technische Universität München and the Helmholtz-Gesellschaft München, for his sixtieth anniversary.

\section{Disclosure/conflict of interest}

The authors declare no conflict of interest.

\section{References}

1 DeMeester SR. Adenocarcinoma of the esophagus and cardia: a review of the disease and its treatment. Ann Surg Oncol 2006;13:12-30.
2 Kubo A, Corley DA. Body mass index and adenocarcinomas of the esophagus or gastric cardia: a systematic review and meta-analysis. Cancer Epidemiol Biomarkers Prev 2006;15:872-878.

3 Jemal A, Siegel R, Ward E, et al. Cancer statistics, 2007. CA Cancer J Clin 2007;57:43-66.

4 Cunningham D, Allum WH, Stenning SP, et al. Perioperative chemotherapy versus surgery alone for resectable gastroesophageal cancer. N Engl J Med 2006;355:11-20.

5 Siewert JR, Lordick F, Ott K, et al. Induction chemotherapy in Barrett cancer: influence on surgical risk and outcome. Ann Surg 2007;246:624-628; discussion 628-631.

6 Boige V, Pignon J, Saint-Aubert B, et al. Final results of a randomized trial comparing preoperative 5-fluorouracil (F)/cisplatin (P) to surgery alone in adenocarcinoma of stomach and lower esophagus (ASLE): FNLCC ACCORD07-FFCD 9703 trial. J Clin Oncol (Meeting Abstracts) 2007;25:4510.

7 Gebski V, Burmeister B, Smithers BM, et al. Survival benefits from neoadjuvant chemoradiotherapy or chemotherapy in oesophageal carcinoma: a meta-analysis. Lancet Oncol 2007;8:226-234.

8 Chang F, Deere H, Mahadeva U, et al. Histopathologic examination and reporting of esophageal carcinomas following preoperative neoadjuvant therapy: practical guidelines and current issues. Am J Clin Pathol 2008;129:252-262.

9 Donington JS, Miller DL, Allen MS, et al. Tumor response to induction chemoradiation: influence on survival after esophagectomy. Eur J Cardiothorac Surg 2003;24:631-636; discussion 636-637.

10 Langer R, Specht K, Becker K, et al. Association of pretherapeutic expression of chemotherapy-related genes with response to neoadjuvant chemotherapy in Barrett carcinoma. Clin Cancer Res 2005;11:7462-7469.

11 Rizk N, Venkatraman E, Park B, et al. The prognostic importance of the number of involved lymph nodes in esophageal cancer: implications for revisions of the American Joint Committee on Cancer staging system. J Thorac Cardiovasc Surg 2006;132:1374-1381.

12 Becker K, Mueller JD, Schulmacher C, et al. Histomorphology and grading of regression in gastric carcinoma treated with neoadjuvant chemotherapy. Cancer 2003; 98:1521-1530.

13 Siewert JR, Stein HJ. Classification of adenocarcinoma of the oesophagogastric junction. Br J Surg 1998; 85:1457-1459.

14 Lordick F, Ott K, Krause BJ, et al. PET to assess early metabolic response and to guide treatment of adenocarcinoma of the oesophagogastric junction: the MUNICON phase II trial. Lancet Oncol 2007;8:797-805.

15 Bader FG, Lordick F, Fink U, et al. Paclitaxel in the neoadjuvant treatment for adeno carcinoma of the distal esophagus (AEG I). A comparison of two phase II trials with long-term follow-up. Onkologie 2008; 31:366-372.

16 Dunne B, Reynolds JV, Mulligan E, et al. A pathological study of tumour regression in oesophageal adenocarcinoma treated with preoperative chemoradiotherapy. J Clin Pathol 2001;54:841-845.

17 Brucher BL, Becker K, Lordick F, et al. The clinical impact of histopathologic response assessment by residual tumor cell quantification in esophageal squamous cell carcinomas. Cancer 2006;106: 2119-2127. 
18 Rosenberg R, Nekarda H, Zimmermann F, et al. Histopathological response after preoperative radiochemotherapy in rectal carcinoma is associated with improved overall survival. J Surg Oncol 2008;97:8-13.

19 Chirieac LR, Swisher SG, Ajani JA, et al. Posttherapy pathologic stage predicts survival in patients with esophageal carcinoma receiving preoperative chemoradiation. Cancer 2005;103:1347-1355.

20 Swisher SG, Hofstetter W, Wu TT, et al. Proposed revision of the esophageal cancer staging system to accommodate pathologic response (pP) following preoperative chemoradiation (CRT). Ann Surg 2005; 241:810-817; discussion 817-820.

$21 \mathrm{Wu}$ TT, Chirieac LR, Abraham SC, et al. Excellent interobserver agreement on grading the extent of residual carcinoma after preoperative chemoradiation in esophageal and esophagogastric junction carcinoma: a reliable predictor for patient outcome. Am J Surg Pathol 2007;31:58-64.

22 Mandard AM, Dalibard F, Mandard JC, et al. Pathologic assessment of tumor regression after preoperative chemoradiotherapy of esophageal carcinoma. Clinicopathologic correlations. Cancer 1994;73:2680-2686.
23 Rizk NP, Venkatraman E, Bains MS, et al. American Joint Committee on Cancer staging system does not accurately predict survival in patients receiving multimodality therapy for esophageal adenocarcinoma. J Clin Oncol 2007;25:507-512.

24 Schneider PM, Baldus SE, Metzger R, et al. Histomorphologic tumor regression and lymph node metastases determine prognosis following neoadjuvant radiochemotherapy for esophageal cancer: implications for response classification. Ann Surg 2005;242:684-692.

25 Berger AC, Farma J, Scott WJ, et al. Complete response to neoadjuvant chemoradiotherapy in esophageal carcinoma is associated with significantly improved survival. J Clin Oncol 2005;23:4330-4337.

26 Brucher BL, Stein HJ, Werner M, et al. Lymphatic vessel invasion is an independent prognostic factor in patients with a primary resected tumor with esophageal squamous cell carcinoma. Cancer 2001;92: 2228-2233.

27 von Rahden BH, Stein HJ, Feith M, et al. Lymphatic vessel invasion as a prognostic factor in patients with primary resected adenocarcinomas of the esophagogastric junction. J Clin Oncol 2005;23:874-879.

Supplementary Information accompanies the paper on Modern Pathology website (http://www.nature.com/ modpathol) 